\title{
Characterization of low-molar-mass polymers by gradient polymer elution chromatography, II. Solubility effects in the analysis of polyester resins under reversed-phase conditions
}

Citation for published version (APA):

Philipsen, H. J. A., de Cooker, M. R., Claessens, H. A., Klumperman, B., \& German, A. L. (1997).

Characterization of low-molar-mass polymers by gradient polymer elution chromatography, II. Solubility effects in the analysis of polyester resins under reversed-phase conditions. Journal of Chromatography, A, 761(1-2), 147162. https://doi.org/10.1016/S0021-9673(96)00807-2

DOI:

10.1016/S0021-9673(96)00807-2

Document status and date:

Published: 01/01/1997

Document Version:

Publisher's PDF, also known as Version of Record (includes final page, issue and volume numbers)

\section{Please check the document version of this publication:}

- A submitted manuscript is the version of the article upon submission and before peer-review. There can be important differences between the submitted version and the official published version of record. People interested in the research are advised to contact the author for the final version of the publication, or visit the $\mathrm{DOI}$ to the publisher's website.

- The final author version and the galley proof are versions of the publication after peer review.

- The final published version features the final layout of the paper including the volume, issue and page numbers.

Link to publication

\footnotetext{
General rights

- You may freely distribute the URL identifying the publication in the public portal. follow below link for the End User Agreement:

www.tue.nl/taverne

\section{Take down policy}

If you believe that this document breaches copyright please contact us at:

openaccess@tue.nl

providing details and we will investigate your claim.
}

Copyright and moral rights for the publications made accessible in the public portal are retained by the authors and/or other copyright owners and it is a condition of accessing publications that users recognise and abide by the legal requirements associated with these rights.

- Users may download and print one copy of any publication from the public portal for the purpose of private study or research.

- You may not further distribute the material or use it for any profit-making activity or commercial gain

If the publication is distributed under the terms of Article $25 \mathrm{fa}$ of the Dutch Copyright Act, indicated by the "Taverne" license above, please 


\title{
Characterization of low-molar-mass polymers by gradient polymer elution chromatography II. Solubility effects in the analysis of polyester resins under reversed-phase conditions
}

\author{
H.J.A. Philipsen ${ }^{a . *}$, M.R. de Cooker ${ }^{b}$, H.A. Claessens ${ }^{c}$, B. Klumperman ${ }^{b}$, A.L. German ${ }^{b}$ \\ 'Océ Netherlands, Research and Development Department (R-AM, location 3B58), P.O. Box 10I, 5900 MA, Venlo. Netherlands \\ "Laboratory of Polymer Chemistry, Eindhoven University of Technology, P.O. Box 513, 5600 MB, Eindhoven, Netheriands \\ "Laboratory of Instrumental Analysis, Eindhoven University of Technology, P.O. Box 513, 5600 MB, Eindhoven, Netherlands
}

Received 23 May 1996; revised 24 September 1996; accepted 25 September 1996

\begin{abstract}
Polyester resins can be characterized by gradient polymer elution chromatography (GPEC) to obtain information on molar mass and chemical composition. The separation can be influenced by size-exclusion, sorption and solubility (precipitation/ re-dissolution) effects. The influence of solubility effects was investigated in this study. Due to pronounced dependencies of molar mass and concentration on cloud points, solubility effects in GPEC were studied under chromatographic conditions, using inert column packings and low polydispersity fractions obtained by size-exclusion chromatography. The investigated system was a reversed-phase system, using water and tetrahydrofuran as solvent/non-solvent (S/NS) combination. Bare silica, non-porous glass and a stainless steel pre-column filter were compared for the use as an inert medium. A non-porous glass column was shown to be the best choice in this respect. By comparison of the results of the various polyester fractions on the glass and $\mathrm{C}_{18}$ column, respectively, it was shown that the separation on $\mathrm{C}_{18}$ is solely determined by sorption effects. The observed correspondence between values of the \%-solvent at the peak-end of the whole, unfractionated polyester on an inert and a sorbing column, alone, is therefore no evidence for solubility governing retention in the high molar mass part of the chromatogram. A comparison with measurements of maximum solubility under static equilibrium conditions of four different polyester fractions in various S/NS combinations revealed that concentrations of the eluting fractions on the glass column are considerably lower than maximum solubility. This can be explained by kinetic effects, influencing re-dissolution.
\end{abstract}

Keywords: Gradient elution; Polymers; Polyesters; Adipic acid polyesters; Isophthalic acid polyesters; Bisphenol-A. dipropoxylated, polyesters

\section{Introduction}

In principle, the characterization of polymers by liquid chromatography can be performed either in the exclusion mode, by size-exclusion chromatography

${ }^{*}$ Corresponding author.
(SEC), or in the sorption mode. In the latter case, mostly gradient elution has to be applied and in practice the separation results from a combination of precipitation/redissolution, adsorption/partitioning and exclusion effects. Since the contribution of these separate mechanisms can be different in each specific case, we prefer the use of the name gradient polymer 
elution chromatography (GPEC) $[1,2]$ rather than existing names such as high-performance precipitation liquid chromatography (HPPLC) [3] or liquid adsorption chromatography (LAC) [4] which a priori assume a certain separation mechanism. Two main fields of application of GPEC include the characterization of copolymers according to their chemical composition distribution [5-7] and the fingerprinting of resins [8-10].

Reversed-phase liquid chromatographic techniques have been used for the characterization of both copolymers and resins [11]. In the first part of this series of papers, we clearly demonstrated the applicability of GPEC under reversed-phase conditions for the characterization of (co)polyester resins according to differences in chemical microstructure [8]. Although it was shown that separation is likely governed by sorption, precipitation/redissolution effects also occur in the investigated system. In this second part we will investigate to what extent precipitation/redissolution effects influence the separation of amorphous (co)polyesters under reversedphase conditions.

Studies to the influence of precipitation effects in polymer chromatography are mostly concerned with the comparison of eluent compositions at the point of elution with cloud points, determined by turbidimetric titrations [12-15]. The relation between the solubility of a polymer in a solvent/non-solvent (S/NS) mixture with volume fraction $\phi$ of the solvent and polymer concentration $c$ is given by [16]

$\phi^{*}=C_{1}+C_{2} \log \left(c^{*}\right)$

in which $C_{1}$ and $C_{2}$ are constants, dependent on the $\mathrm{S} / \mathrm{NS}$ system, the temperature and the molar mass. $c^{*}$ and $\phi^{*}$, respectively, indicate values at the cloud point. Therefore, care must be taken that concentrations in both chromatography and cloud point titration are comparable. The dependence of the volume fraction of solvent at the cloud point, $\phi_{\mathrm{S}}^{*}$, on the molar mass is given by [17]

$\phi_{\mathrm{S}}^{*}=C_{3}+C_{4} M^{-0.5}$

where $C_{3}$ and $C_{4}$ are constants, dependent on the S/NS system and on temperature. From Eq. (2) it is obvious that especially for relatively low molar mass resins, $\phi_{\mathrm{S}}^{*}$ for the different oligomeric species will strongly depend on molar mass. Even for polyester resins with relatively low polydispersity (approximately 2), a single determination of the cloud point of the complete resin can obviously not be used for comparison with chromatographic data to account for the retention mechanism. Such a comparison is further hampered by the fact that the concentration dependence of the cloud point increases with decreasing molar mass, which results in lower values of $C_{2}$ in Eq. (1).

An alternative method by which sorption and precipitation effects can be separately controlled, was presented by Glöckner who used so-called sudden transition (ST) gradients for this purpose [18-20]. After injection into a strong non-solvent, the eluent composition is rapidly changed by addition of a solvent of moderate polarity, which causes the precipitated polymer to redissolve. However, the S/NS composition is changed in such a way that the sample is still retained by sorption forces. Finally, a chromatographically strong eluent ("displacer"), which is not necessarily a solvent, is added, causing the polymer to elute. By comparison of these results with a true S/NS gradient, the influence of redissolution effects can be determined. The application of ST gradients for low molar mass polymers, however, is restricted by the fact that cloud points of different molar mass fractions will vary over a wide composition range. Therefore, especially under reversed-phase conditions where interaction forces with the stationary phase are weak, mostly no composition can be found that will completely redissolve the resin and simultaneously causes complete retention.

Thus, solubility effects of low-molar-mass polymers under reversed-phase conditions can at best be studied, under chromatographic conditions, using inert column packings and low polydispersity polymer fractions. Therefore, a column filled with spherical glass beads, a bare silica column and a precolumn filter were tested for the use as an inert medium for polyester resins. A (co)polyester resin which has also been used for earlier experiments [8], was separated into several low polydispersity fractions by SEC. The obtained fractions were subjected to GPEC on several inert media and results were compared with a $\mathrm{C}_{18}$ column, thus showing whether or not the absence of sorption influences the separation. The used S/NS system, tetrahydrofuran (THF)-water, was the same as used in the previous 
study [8]. Furthermore, for three different polyester fractions, results on the glass column were compared with maximum solubility measurements in various S/NS compositions under static equilibrium conditions. This evaluation will clearly show the importance of dissolution kinetics. Although these effects were not likely to influence the final separation result of the investigated amorphous polyester on $\mathrm{C}_{18}$, in a future paper we will show the importance of redissolution effects for crystalline polyesters [21].

\section{Experimental}

\subsection{Polymer sample and narrow disperse fractions}

The polymer sample used is a co-polyester resin consisting of adipic acid (A), isophtalic acid (I) and dipropoxylated bisphenol-A (D) (sample PE1 from [8]). The molar ratio A:I:D is approximately $0.12: 0.38: 0.50$ (NMR), the acid number is $20 \mathrm{mg}$ $\mathrm{KOH} / \mathrm{g}$ and the polystyrene equivalent molar masses as determined by SEC are 3500 (number-average molar mass, $M_{\mathrm{n}}$ ) and 7900 (weight-average molar mass, $M_{\mathrm{w}}$ ), respectively. For more detailed information on the composition and characterization of the polyester sample we refer to [8].

Low polydispersity fractions were obtained by SEC. A $25 \mathrm{mg} / \mathrm{ml}$ solution was injected 200 times on the SEC system described in Ref. [8], using an injection volume of $200 \mu \mathrm{l}$. The total distribution which eluted in about 10 min (see Fig. 5 of Ref. [8]) was separated into 10 fractions of $1 \mathrm{~min}$, using a Gilson (Villiers-le-Bel, France) Model 203 fraction collector. The fractions were dried under nitrogen and the amount was determined gravimetrically. A part of each fraction was redissolved in THF up to a concentration of $0.4 \mathrm{mg} / \mathrm{ml}$ and $200 \mu \mathrm{l}$ was reinjected on the SEC system to determine the respective polydispersity values. For gradient elution experiments where the exact injected amounts had to be known, concentrations of these solutions were carefully determined by HPLC, as described in Ref. Section 2.7 .

\subsection{Solvents}

The solvents used for HPLC and solubility experiments were water, LiChrosolv quality from Merck
(Darmstadt, Germany) and tetrahydrofuran (THF), HPLC grade from Rathburn (Brunschwig Chemie, Amsterdam, Netherlands). To both solvents, $200 \mu \mathrm{l}$ acetic acid, analytical-regent quality from Merck, per litre was added. For HPLC, the solvents were constantly sparged with helium $(20 \mathrm{ml} / \mathrm{min})$. All solvent mixtures were made by volumetric mixing by the HPLC pump, no premixes were used.

\subsection{Columns}

The columns used were a Novapak- $\mathrm{C}_{18}$ column (Waters, Milford, MA, USA; $d_{\mathrm{p}}=4 \mu \mathrm{m}$, pore size 60 $\AA, 150 \times 3.9 \mathrm{~mm}$, plate count $/ \mathrm{m}$ ca. 80000 ), a Resolve silica column (Waters, $d_{\mathrm{p}}=5 \mu \mathrm{m}$, pore size $90 \AA, 150 \times 3.9 \mathrm{~mm}$, plate count $/ \mathrm{m}$ ca. 55000$)$ and a PL-gel styrene-divinylbenzene (Polymer Labs., Shropshire, UK, $d_{\mathrm{p}}=5 \mu \mathrm{m}$, pore size $100 \AA$, $600 \times$ $7.5 \mathrm{~mm}$, plate count $/ \mathrm{m}$ ca. 60000 ) which was used for solubility experiments (see Section 2.7). Furthermore, for gradient elution experiments a column $(150 \times 4.6 \mathrm{~mm})$ was dry-packed with non-porous glass beads, diameter $40-60 \mu \mathrm{m}$ (Phase Separations, UK, part No. 750138). The performance of this column was tested by the injection of $5 \mu$ l toluene in water. The asymmetry of the resulting peak was found to be 1.2. The dead volume $\left(V_{0}\right)$ for each column was determined not only by the injection of a low molar mass solute (toluene) but also for each individual polyester fraction. The thus found values were used in further calculations to correct for SEC effects. For the silica and the $\mathrm{C}_{18}$ column these values were found to vary between $0.89-1.36 \mathrm{ml}$ and 0.78-1.07 ml, respectively. For the glass column, all values equalled $1.00 \mathrm{ml}$.

All columns were connected by a $0.02 \mathrm{~cm}$ (I.D.) tubing of about $50 \mathrm{~cm}$ to the injector, which was led through the column thermostat to ensure the sample to reach the right temperature before entering the column. For all experiments a stainless steel in-line pre-column filter (Waters, part No. 084560) was used.

\subsection{HPLC equipment}

All HPLC experiments were performed using a Waters 600E 4 solvent gradient pump and a 717 autosampler or a 715 WISP from Waters. The 
detector was a variable-wavelength detector, Waters, type 484 or a Jasco (Tokyo, Japan) type 975 , which was set at $277 \mathrm{~nm}$. The column temperature was controlled using a thermostat type Mistral from Spark-Holland (Emmen, Netherlands). Chromatograms were recorded using the Baseline- 815 system from Waters.

\subsection{Gradient elution experiments}

Gradient elution was performed as follows. A gradient was run from water-THF (both containing $0.02 \%, \mathrm{v} / \mathrm{v}$, acetic acid) $100: 0$ to $0: 100$ in $33.3 \mathrm{~min}$. $(3 \% / \mathrm{min})$. After running each gradient, the system was reset to initial conditions in one minute, followed by pumping $15 \mathrm{ml}$ of the starting eluent composition to re-equilibrate the column. Prior to the analysis of the samples, two blank gradients were run. Initial conditions were chosen at $100 \%$ water because small parts of the low-molar-mass fractions were suspected to be already soluble under these conditions. Starting at higher amounts of THF would cause significant amounts to elute unretained on inert columns which would hamper a good comparison with elution on $\mathrm{C}_{18}$. Although it is known that initial conditions of $100 \%$ water in reversed-phase HPLC can sometimes lead to bad reproducibility, no such problems were encountered during our experiments. All gradients were started at the moment of injection. The gradient performance of the pump (linearity and reproducibility) was checked by running gradients from methanol to methanol $+0.1 \%(\mathrm{v} / \mathrm{v})$ acetone. The linearity was found to be excellent, thus allowing an easy calculation of the eluting eluent composition at each retention time. The system hold-up volume was also determined from these experiments and was found to be $4.0 \mathrm{ml}$. Unless indicated otherwise, all measurements were carried out at $21^{\circ} \mathrm{C}$.

\subsection{Cloud point titrations}

Cloud points of the unfractionated polyester and polyester fractions were determined by visual observation at room temperature $\left(21^{\circ} \mathrm{C}\right)$. For the polyester fractions, $0.5 \mathrm{ml}$ of a solution in THF was brought into a micro titration vial equipped with a magnetic stirrer bar [22]. Concentrations were taken such that the final concentration in the cloud point was approximately $1.5 \mathrm{mg} / \mathrm{ml}$. By means of a $100 \mu \mathrm{l}$ syringe, small portions of the non-solvent (water) were added until the solution became turbid. The point at which turbidity did not disappear after stirring was defined as the cloud point. \%-Solvent at this point was determined gravimetrically. All cloud points were determined three times. The standard deviation of the three measurements did not exceed $0.3 \%$ solvent, except for the very-low-molar-mass fractions where somewhat larger values were found.

\subsection{Determination of solubility}

The sample preparation for the determination of the solubility as function of the S/NS ratio was as follows. From a solution of $30 \mathrm{mg} / \mathrm{ml}$ (THF) of a respective polyester fraction, $100 \mu \mathrm{l}$ was taken with a syringe and transferred into a $4 \mathrm{ml}$ vial. After drying under nitrogen, water and THF, both containing $0.02 \%(\mathrm{v} / \mathrm{v})$ acetic acid were added to a volume of $1.0 \mathrm{ml}$, such that a desired S/NS ratio was obtained. All amounts were determined by mass. After thoroughly shaking for $10 \mathrm{~min}$, the vial was put in a temperature controlled water bath at $21^{\circ} \mathrm{C}$. After equilibration for one night, the suspension was centrifuged for $6 \mathrm{~min}$ at $3000 \mathrm{~g}$. From the obtained supernatant, $300 \mu \mathrm{l}$ was carefully taken with a syringe and centrifuged again. Finally, a small amount of the clear solution was injected on a HPLC system, to determine the polyester concentration. This method resembles that of other workers [23], but modifications had to be made due to the much lower molar masses of our polymers.

It is known that determination of solubility of polymers from the dry state, especially in the case of high-molar-mass polymers can provide different results from the more accurate approach of precipitation from solution [24]. The former procedure was preferred in our case, however, since a comparison of both methods revealed that for our low-molarmass polymers in the latter method too high values were obtained in the low concentration range $(<0.05$ $\mathrm{mg} / \mathrm{ml}$ ) probably due to slow precipitation [22]. For higher concentrations, identical values for both methods were obtained.

For the HPLC measurements, a PL-gel styrenedivinylbenzene column (see Section 2.3) was used, 
which was held at $21^{\circ} \mathrm{C}$. The eluent was THF containing $1 \%(\mathrm{v} / \mathrm{v})$ acetic acid. The injection volume was in the range 5-100 $\mu \mathrm{l}$, depending on the (estimated) polyester concentration. All solutions were at least injected twice. The system was calibrated using three independent solutions of the unfractionated polyester with known concentrations. From earlier experiments it was known that the extinction coefficient does not show any significant dependence on molar mass at the used detection wavelength [8], thus allowing for the chosen calibration procedure.

\section{Results and discussion}

The "poiystyrene equivalent" molar masses, polydispersity values and amounts of the low dispersity polyester fractions obtained by SEC, are given in Table 1. Most of the measured polydispersity values are close to 1.1 , thus making the fractions suitable for solubility studies. Since solubility and retention in GPEC both strongly depend on molar mass (Eq. (2)), polydispersity values must be as low as possible to enable a meaningful comparison between solubility under equilibrium conditions and chromatographic conditions, respectively.

As could be expected for low-molar-mass polymers, the influence of concentration on the cloud point composition (CPC) is relatively large. This is demonstrated in Fig. 1 where the CPC of the unfractionated polyester is plotted as a function of its concentration. The variation of CPC with temperature, which was also investigated for the unfraction- ated polyester, was found to be approximately $0.15 \% /{ }^{\circ} \mathrm{C}[22]$. Since the concentration and temperature dependencies increase towards lower molar masses, this situation would even be worse for the low-molar-mass fractions.

Therefore, for comparison of CPC values with chromatographic results, care must be taken that concentrations in both cases are identical. This approach, which can be used for high-molar-mass polymers [12] would fail in our case, due to the extreme molar mass dependence of retention in the low-molar-mass range. Although the polydispersity of the fractions in our study is low, they still consist of a mixture of related substances differing in molar mass and chemical composition, giving rise to different elution volumes (see for instance Fig. 2, $\mathrm{C}_{18}$ column). This results in a considerable chromatographic dissolution, that would have to be compensated by the injection of very high concentrations which might cause redissolution problems or chromatographic overloading thus influencing the elution behaviour.

Therefore, solubility effects can at best be studied under non-sorption chromatographic conditions, thus necessitating the availability of inert column packings. The use of normal-phase packings, e.g., bare silica, under reversed-phase conditions, has been suggested for this purpose $[15,25]$. Non-porous glass beads were taken into account as a possible alternative.

Although it might be expected that acidified water would strongly suppress column's residual silanol groups, it is known that, even under such circumstances, in certain cases silica as well as glass can be

Table 1

Polystyrene equivalent molar masses, polydispersity values and amounts of low polydispersity polyester fractions obtained by SEC

\begin{tabular}{ccccc}
\hline Fraction number & $M_{\mathrm{n}}$ & $M_{\mathrm{w}}$ & Polydispersity & Obtained amount (g) \\
\hline 1 & 33300 & 38000 & 1.14 & 1.07 \\
2 & 21000 & 22500 & 1.07 & 0.0075 \\
3 & 12000 & 12800 & 1.07 & 0.0707 \\
4 & 7300 & 7800 & 1.05 & 0.1506 \\
5 & 4200 & 4400 & 1.04 & 0.2474 \\
6 & 2500 & 2600 & 1.07 & 0.1568 \\
7 & 1500 & 1600 & 1.18 & 0.0870 \\
8 & 960 & 1130 & 1.45 & 0.0564 \\
9 & 650 & 940 & 1.93 & 0.0202 \\
10 & 401 & 775 & & 0.00120 \\
& & & & $0.8102($ total amount) \\
\hline
\end{tabular}




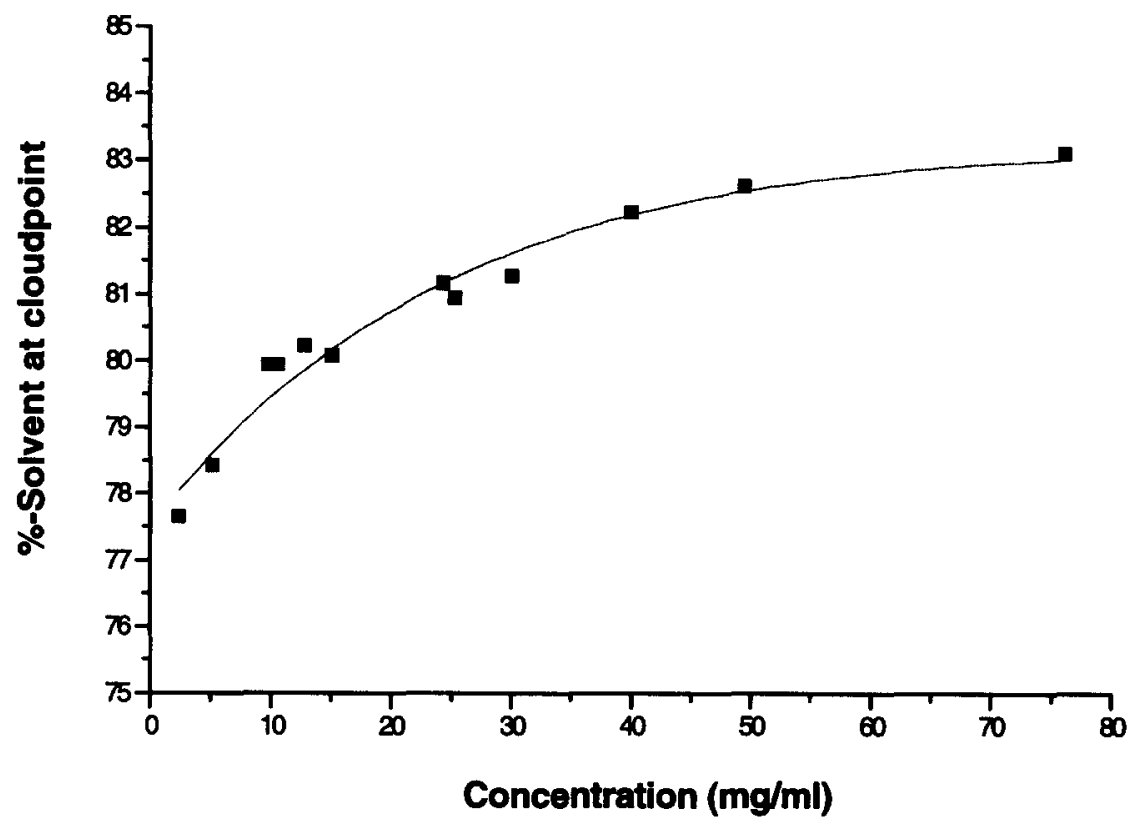

Fig. 1. Influence of the polyester concentration on the cloud point composition. Temperature $=21^{\circ} \mathrm{C}$, solvent $/$ non-solvent $=\mathrm{THF}-\mathrm{water}$.

retentive [26]. Therefore, the elution behaviour of the polyester fractions was tested in THF containing $0.02 \%(\mathrm{v} / \mathrm{v})$ acetic acid and in THF-water (85:15, $\mathrm{v} / \mathrm{v})+0.02 \%$ acetic acid, which about equals the S/NS composition at which the last part of the polyester elutes [8]. In THF, for both columns parts of the injected fractions were found to elute after the column dead volume $\left(V_{0}\right)$, indicating adsorptive interactions. In the THF-water mixture however, all fractions completely eluted at $V_{0}$ in the case of non-porous glass and before $V_{0}$ in the case of silica, due to SEC effects. Complete elution was further confirmed by comparison of peak areas with results obtained on the $C_{18}$ column, which is known to completely elute the polyester [8]. Since higher water contents might even better mask residual silanol groups, both glass and bare silica were assumed to be inert in the set-up of our experiments.

The use of columns, packed with small metal particles was also considered, although it is known that even stainless steel is not completely inert in all cases [27]. Since the packing of these columns was hampered by the high specific mass of the materials [22], in this respect only the use of a stainless steel pre-column filter was studied.

Consequently, the retention behaviour of low dispersity polyester fractions was compared using a $\mathrm{C}_{18}$ column, a non-porous glass column, a silica column and a stainless steel pre-column filter. The injected sample amount was taken as low as possible, since it is known that in the case of solubility effects governing retention, retention times may shift to higher values with increasing sample load [15]. Due to interference with retention differences caused by different extents of column interactions, this might hamper comparison of results from different columns. An injected mass of $2 \mu \mathrm{g}$ proved to be a reasonable compromise between sample load and detectability. Since gradient time was found to have only a slight influence on the retention characteristics (see also Fig. 7b), for reasons of detectability and analysis time a steepness of $3 \% / \mathrm{min}$. was chosen.

In Fig. 2, results of the respective columns are shown. Fractions were injected at least twice on each column and results werc found to be highly reproducible. The retention times in all chromatograms were corrected for the system hold-up time and the column dead time, according to

$t_{\mathrm{r}(\text { corrected })}=t_{\mathrm{r}}-t_{\mathrm{s}}-t_{\mathrm{sec}}$

where $t_{\mathrm{r}}$ is the retention time, $t_{\mathrm{s}}$ is the system 

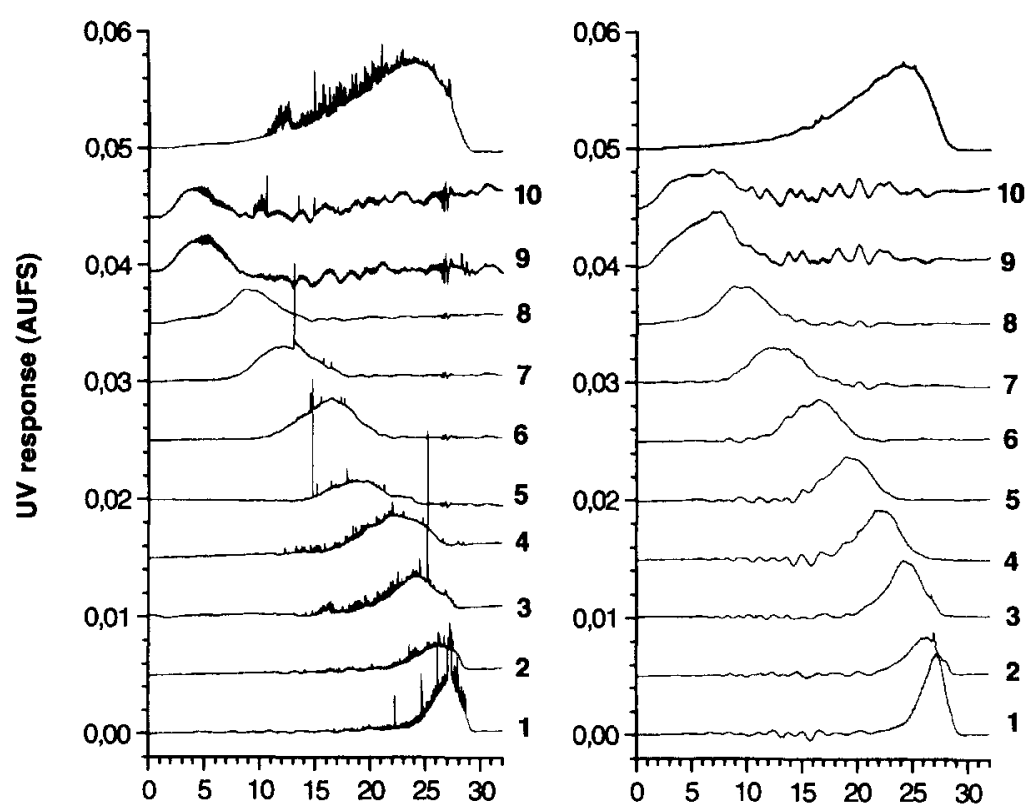

Time (min.)

a

Time (min.) b
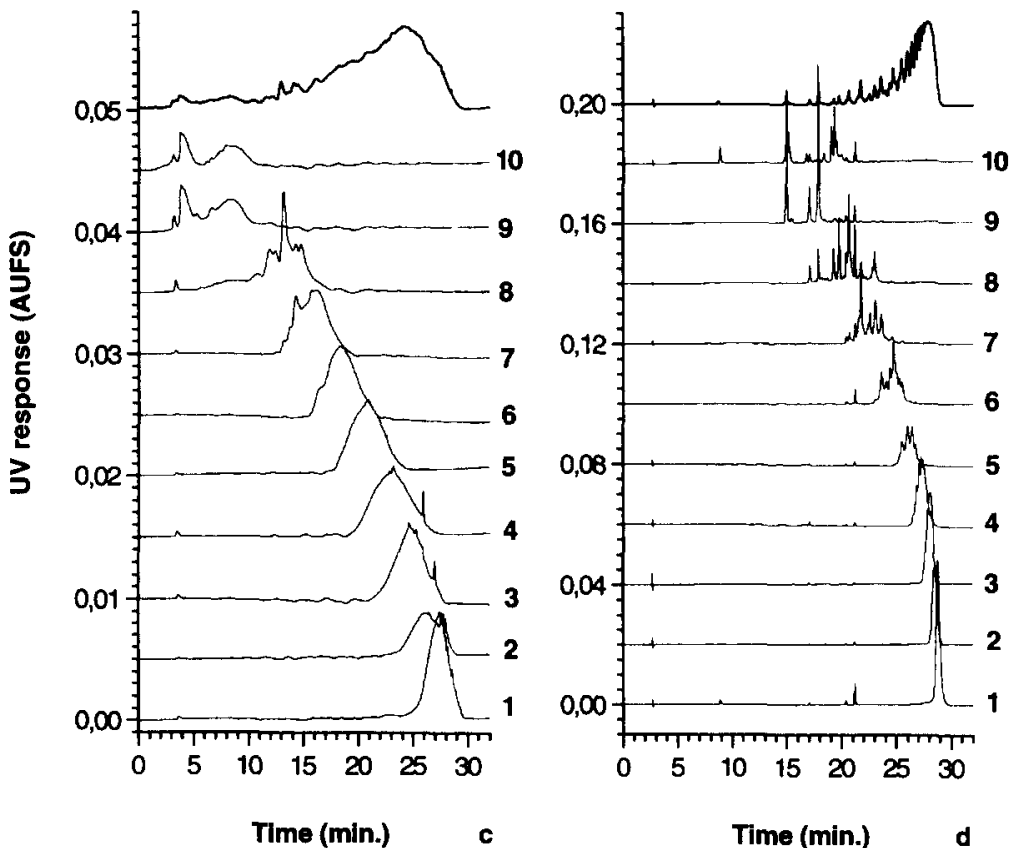

Fig. 2. Elution of low polydispersity fractions (1-10) (Table 1) and unfractionated polyester (upper curves) on different columns. (a) Pre-column filter, (b) glass column, (c) silica column, (d) $\mathrm{C}_{18}$ column. Conditions: sample concentration, $0.4 \mathrm{mg} / \mathrm{ml}$ in THF; eluent, THF-water $(0: 100)$ to $(100: 0)$ in $33.3 \mathrm{~min}$; flow, $1.0 \mathrm{ml} / \mathrm{min}$; temperature, $21^{\circ} \mathrm{C}$; injection, $5 \mu \mathrm{l}$; detection, UV at $277 \mathrm{~nm}$. Chromatograms time-corrected according to Eq. (3). 
hold-up time and $t_{\mathrm{sec}}$ is the column dead volume $\left(t_{0}\right)$ for the respective fraction. The latter was found to depend on the molar mass due to SEC effects (see Section 2.3).

Due to insufficient mixing with the eluent, part of the injected sample which was dissolved in THF eluted unretained from the glass column and the guard filter. Since this cannot be observed in the time-corrected chromatograms in Fig. 2, an example of an uncorrected chromatogram is shown in Fig. 3. Especially for the fractions with lowest molar masses, this effect was excessive, thus giving rise to a low signal-to-noise ratio, as can be observed in the chromatograms of fractions 9 and 10. By measuring peak areas of the unretained peak and the normal eluting peak, the amount of sample which eluted unretained, was calculated. Results are shown in Table 2. It can be observed that for both columns the effect was found to increase upon decreasing molar masses. Since these breakthrough effects were not observed for the silica and $\mathrm{C}_{18}$ column and were worse for the pre-column filter as compared to the glass column, they may be caused by a reduction in the surface area available for precipitation. Furthermore, the columns used will also exhibit significantly different internal mixing due to the difference in particle diameter, which can also influence the
Table 2

Fraction of the sample eluting unretained due to breakthrough on the pre-column filter and the glass column

\begin{tabular}{cll}
\hline Fraction number & Pre-column filter & Glass column \\
\hline 1 & $0.14 \pm 0.04^{\mathrm{a}}$ & $0.08 \pm 0.04^{\mathrm{a}}$ \\
2 & 0.26 & 0.14 \\
3 & 0.14 & 0.06 \\
4 & 0.19 & 0.06 \\
5 & 0.24 & 0.06 \\
6 & 0.22 & 0.10 \\
7 & 0.25 & 0.13 \\
8 & 0.44 & 0.34 \\
9 & 0.84 & 0.57 \\
10 & 0.86 & 0.45 \\
\hline
\end{tabular}

Maximum deviation between average and maximum value of duplicate measurements.

elution behaviour of polymers and the occurrence of breakthrough effects [28].

The high frequency noise in the chromatograms from the pre-column filter is probably caused by parts of the sample that were not completely redissolved and therefore eluted as small polymer particles, causing light scattering in the UV detector. The effect becomes worse when sample load is increased (result not shown here) thus supporting this assumption. Since these effects are absent in results from the glass column, it is obvious that due to

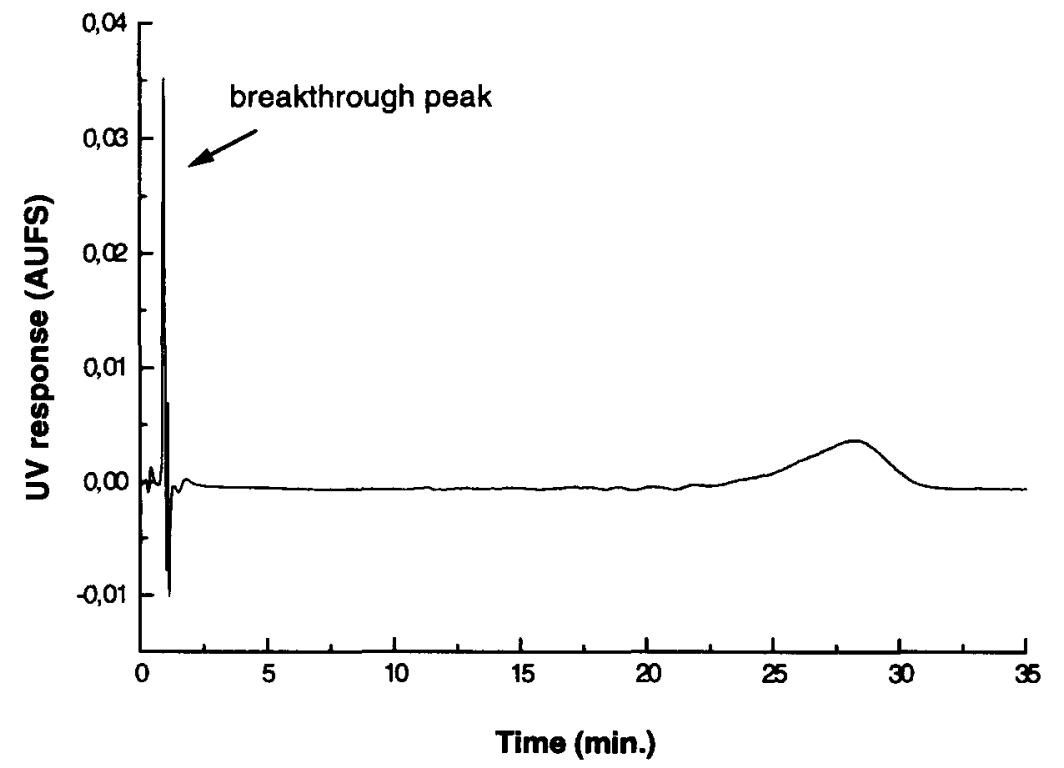

Fig. 3. Blank-corrected chromatogram of polyester fraction 3 on the glass column, before time correction. GPEC conditions: see Fig. 2 . 
mixing effects in the column, the sample is better able to redisolve.

To enable a more accurate comparison of results from the different columns, from all chromatograms, the \%-solvent at the beginning, maximum, and end of the distribution were determined, using Eq. (4),

$\%$ Solvent $=\left(\left(t_{\mathrm{r}}-t_{\mathrm{s}}-t_{\mathrm{sec}}\right) / t_{\mathrm{g}}\right) \cdot 100$ where $t_{\mathrm{r}}$ is the retention time, $t_{\mathrm{s}}$ is the system hold-up time, $t_{\mathrm{sec}}$ is the column dead volume for the respective fraction and $t_{\mathrm{g}}$ is the gradient time.

All values were determined as the average value from two injections. The maximum deviation between the average and the lowest or highest value of a duplicate measurement was found to be about $1.5 \%$-solvent for peak starts and peak ends and

Table 3

\%-solvent at peak start, peak top and peak end of low polydispersity polyester fractions on the investigated columns and in the cloud point

\begin{tabular}{|c|c|c|c|c|c|}
\hline \multirow{2}{*}{$\begin{array}{l}\text { Fraction } \\
\text { number }\end{array}$} & \multicolumn{5}{|l|}{$\%$-Solvent ${ }^{\mathrm{a}}$} \\
\hline & $\begin{array}{l}\text { Pre-column } \\
\text { filter }\end{array}$ & $\begin{array}{l}\text { Glass } \\
\text { column }\end{array}$ & $\begin{array}{l}\text { Silica } \\
\text { column }\end{array}$ & $\begin{array}{l}\mathrm{C}_{18} \\
\text { column }\end{array}$ & $\begin{array}{l}\text { Cloud } \\
\text { point }\end{array}$ \\
\hline \multicolumn{6}{|c|}{ Peak start } \\
\hline 1 & 63.5 & 66.0 & 68.0 & 82.5 & \\
\hline 2 & 57.5 & 64.0 & 67.0 & 82.0 & \\
\hline 3 & 54.0 & 55.0 & 63.0 & 80.0 & \\
\hline 4 & 47.0 & 50.0 & 55.5 & 76.5 & \\
\hline 5 & 38.5 & 38.5 & 50.0 & 73.0 & \\
\hline 6 & 29.0 & 28.5 & 44.0 & 63.0 & \\
\hline 7 & 16.0 & 10.5 & 34.0 & 60.0 & \\
\hline 8 & 4.5 & 1.5 & 11.0 & 50.5 & \\
\hline 9 & 1.0 & 0.0 & 5.0 & 43.5 & \\
\hline 10 & 1.0 & 1.0 & 3.5 & 25.5 & \\
\hline \multicolumn{6}{|c|}{ Peak top } \\
\hline 1 & 82.0 & 82.0 & 82.0 & 85.5 & \\
\hline 2 & 77.0 & 80.0 & 80.5 & 85.0 & \\
\hline 3 & 73.0 & 73.0 & 74.0 & 83.5 & \\
\hline 4 & 66.5 & 66.0 & 70.0 & 81.5 & \\
\hline 5 & 58.5 & 58.5 & 62.5 & more than 1 peak & \\
\hline 6 & 49.5 & 49.0 & 55.0 & & \\
\hline 7 & 37.5 & 38.5 & 48.5 & & \\
\hline 8 & 27.0 & 28.0 & 39.5 & & \\
\hline 9 & 15.5 & 22.5 & 25.4 & & \\
\hline 10 & 13.0 & 18.5 & 24.5 & & \\
\hline \multicolumn{6}{|c|}{ Peak end } \\
\hline 1 & 88.5 & 89.0 & 89.5 & 89.5 & 80.5 \\
\hline 2 & 86.5 & 87.0 & 87.0 & 88.0 & 79.5 \\
\hline 3 & 85.0 & 86.0 & 85.0 & 86.5 & 75.5 \\
\hline 4 & 81.0 & 81.5 & 83.5 & 86.5 & 72.0 \\
\hline 5 & 74.5 & 73.5 & 76.0 & 83.5 & 66.0 \\
\hline 6 & 65.5 & 63.5 & 67.0 & 81.5 & 60.0 \\
\hline 7 & 55.5 & 58.5 & 60.0 & 80.0 & 51.0 \\
\hline 8 & 45.0 & 45.5 & 55.5 & 72.5 & 43.5 \\
\hline 9 & 26.5 & 38.5 & 39.5 & 65.5 & \\
\hline 10 & 25.5 & 35.0 & 33.5 & 66.0 & \\
\hline
\end{tabular}

"All values are average values of two determinations. Maximum deviation between average and maximum value of duplicate measurements: $1.5 \%$-solvent for peak starts and peak ends and $0.5 \%$ for peak tops and cloud points. 
$0.5 \%$-solvent for peak tops. Results are shown in Table 3.

From Fig. 2 and Table 3 it is clear that results on the glass column and the pre-column filter are roughly comparable. Since peak start and peak ends cannot be determined unambiguously in all cases, most differences are within experimental error of determination. Fractions 9 and 10 seem to elute somewhat later from the glass column as compared to the pre-column filter. This can probably be ascribed to the relatively high amounts of sample eluting unretained from the pre-column filter (Table 2). Since the fraction eluting in the gradient is significantly lower, elution is shifted to earlier retention times. Peak tops, which are more accurate to determine coincide in the other cases, which confirms that the retention on the glass column under experimental conditions, is only determined by solubility effects. This is further confirmed by the fact that retention time of the peak top increases when sample load is increased, which is illustrated in Table 4. When sorption effects would contribute to the separation, no dependency or decreasing retention times due to overloading should have been observed [16,29].

The use of a silica column gives rise to additional retention as compared to the pre-column filter, which is generally more manifest for the lower molar mass components as can be concluded from Fig. 2. This is somewhat surprising, since no retention due to sorption effects was observed in isocratic experiments using a THF-water composition of $85: 15$. The additional retention at higher water contents can probably be ascribed to solvophobic effects due to minor affinity of polyester towards the mobile phase at the point of re-dissolution. The different behaviour of silica as compared to non-porous glass was also observed by other workers [15]. Due to the large differences in surface area, this can probably be attributed to the differences in phase ratio $\left(V_{\mathrm{s}} / V_{\mathrm{m}}\right)$ which, in the case of glass, will be orders of magnitude lower. Furthermore, differences in chemical composition and silanol activity of the surface may also contribute to a different retention behaviour.

From the above discussion it is obvious that a bare-silica column cannot be used as an inert column packing for our experiments. The use of a nonporous glass column seems to be the best choice in this respect, since problems caused by breakthrough or insufficient re-dissolution are lower as compared to the pre-column filter!

Furthermore, it must be mentioned that the polyesters used here, are relatively extreme samples due the highly polar carboxylic end groups. Since non-porous glass with a very low phase ratio can be used even for these samples without sorption effects occurring, it is probable that this approach for studying solubility effects in polymer chromatography under reversed-phase conditions can be applied to a rather wide range of polymers.

A comparison with results on the $\mathrm{C}_{18}$ column (Table 3), immediately reveals that separation on $\mathrm{C}_{18}$ is dominated by sorption effects, which again is more manifest for the lower-molar-mass components. Furthermore, especially for the low-molarmass fractions a distinct separation into different peaks can be observed. This is not the case when a glass column is used and must therefore be the result of sorption. Furthermore, from a comparison of peak

Table 4

Influence of sample load on the elution of low polydispersity polyester fractions on the glass column

\begin{tabular}{lclll}
\hline Fraction number & $\begin{array}{l}\text { Injected amount } \\
(\mu \mathrm{g})\end{array}$ & $\begin{array}{l}\text { Peak start } \\
(\%-\mathrm{S})\end{array}$ & $\begin{array}{l}\text { Peak top } \\
(\%-\mathrm{S})\end{array}$ & $\begin{array}{l}\text { Peak end } \\
(\%-\mathrm{S})\end{array}$ \\
\hline 2 & 2 & $64.4 \pm 1.5^{\mathrm{a}}$ & $69.0 \pm 0.5^{\mathrm{a}}$ & $85.5 \pm 1.5^{\mathrm{a}}$ \\
& 15 & 64.4 & 69.8 & 71.2 \\
4 & 2 & 47.5 & 67.0 & 79.5 \\
6 & 15 & 47.4 & 69.1 & 59.7 \\
\end{tabular}

${ }^{a}$ Maximum deviation between average and maximum value of duplicate measurements. 
starts (Table 3 ), it is obvious that elution on a $C_{18}$ column occurs at a much higher \%-solvent of the eluent as compared to the glass column. Since solubility capacity is high enough in such a case, re-dissolution effects, although present in the used system, will not dominate the final separation on a $\mathrm{C}_{18}$ column. This is in accordance with our previous results $[8]$ for which no noticeable retention difference with increasing sample load was found. Obviously, the influence of sample load on a $\mathrm{C}_{18}$ column, is determined by the sorption capacity of the column, rather than the solubility capacity of the eluent.

From the present results, however, it is also obvious that the retention difference for a separation which is controlled by solubility effects as compared to a separation based on sorption, decreases for increasing molar mass. This can be observed from Fig. 4 in which $\%$-solvent at the peak-ends $\left(\phi_{\mathrm{e}}\right)$ on different columns are compared.

This can be explained as follows. During gradient elution, the increase of \%-solvent will both influence solubility and sorption. The retention factor, $k^{\prime}$, which expresses the contribution of sorption is initially very high and gradually decreases until it drops below a value at which the sample starts migrating [30]. Therefore, in the case of gradient elution generally an average retention factor, $K_{\mathrm{av}}$ is used, which is given by [31]:

$K_{\mathrm{av}}=\left(t_{\mathrm{g}} F / 1.15 V_{\mathrm{m}} \Delta \phi S\right)$

in which $t_{\mathrm{g}}$ is the gradient time (min), $F$ is flow-rate $(\mathrm{ml} / \mathrm{min}), V_{\mathrm{m}}$ is the column dead volume, $\Delta \phi$ is the change in the volume fraction of the strong solvent during the gradient and $S$ is an isocratic parameter determined by the strong solvent and the sample compound which is defined as $-d\left(\ln k^{\prime}\right) / d \phi . S$ further depends on molar mass, for which it is sometimes found $[29,30]$

$S=a M^{b}$

For polystyrenes under reversed-phase conditions, using a THF-water system values of $a=0.22$ and $b=0.5$ are reported $[29,30]$.

Therefore, for increasing molar masses, $K_{\mathrm{av}}$ will decrease to a very low value. The range of solvent composition over which polymer solutes migrate becomes very narrow, thus resulting into narrow peaks. Physically this also means, that after reaching the point at which migration starts, the sample will elute almost unretained without significant distribution into the stationary phase. Retention in reversed-

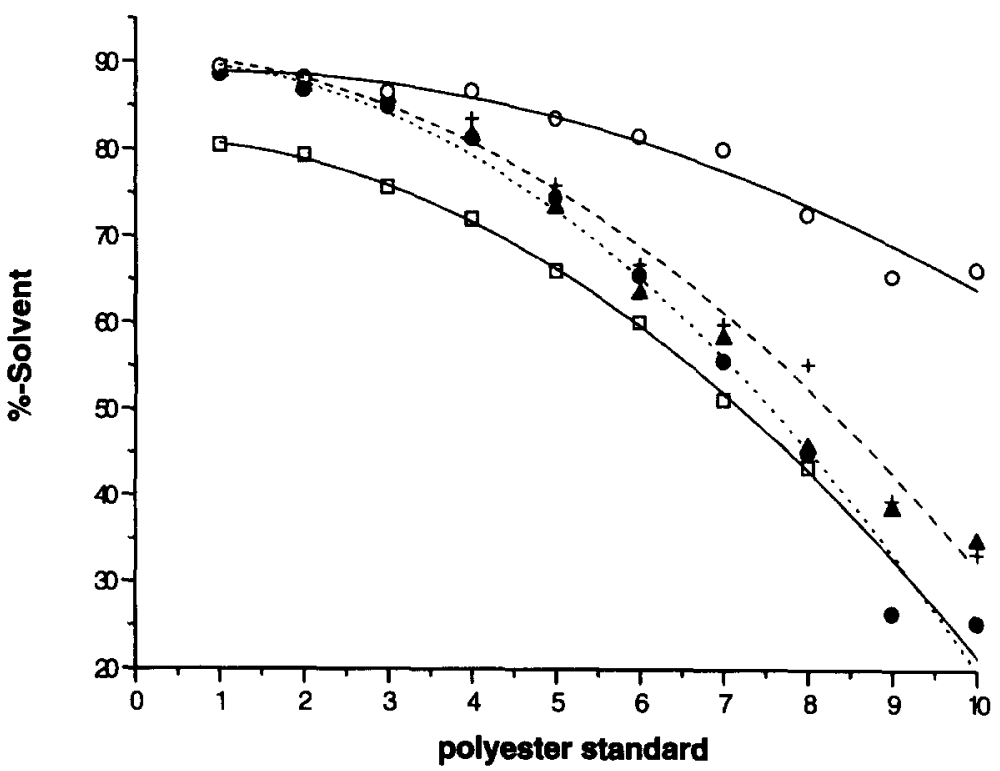

Fig. 4. Cloud points and peak ends on different columns, of the respective polyester fractions. ( $\square$ ) Cloud point; silica; (O) $\mathrm{C}_{18}$. GPEC conditions: see Fig. 2. 
phase systems is to a large extend determined by interactions between the sample and mobile phase, which are the same interactions that determine solubility. Thus, for increasing molar masses, especially on reversed-phase systems, the retention difference for a separation dominated by solubility as compared to a separation governed by sorption, decreases.

Therefore, the finding of corresponding values of $\phi_{\mathrm{e}}$ on an inert and a sorbing column, which can also be found for the unfractionated polyester in this study (Fig. 2) is certainly no evidence for solubility dominating retention in the high-molar-mass part of the chromatogram. Experiments using narrow disperse fractions as shown in this study, a careful study on the effect of sample load, as suggested by other workers [29] or measurements under isocratic conditions in the narrow S/NS range over which a polymer solute migrates [30], are necessary to discriminate between solubility and sorption.

In Fig. 4, also the cloud points of the individual fractions are plotted. As might be expected, because of the low molar masses the cloud points do not coincide with $\phi_{\mathrm{c}}$ values on the inert glass column. It is surprising, however, to see that \%-solvent in the cloud points is considerably lower than $\phi_{e}$, whereas concentrations under chromatographic conditions are much lower due to dilution effects. To further investigate this effect, a comparison was made between measurements of maximum solubility of four different polyester fractions in varying $\mathrm{S} / \mathrm{NS}$ compositions and the concentration profiles of the eluting fractions on the glass column. In Fig. 5, results of measurements of solubility under static conditions are shown.

As could be expected from Eq. (1), a near linear dependence is found between $\log$ (concentration) and $\%$-solvent in the high concentration range. At low concentrations, deviations from this dependence occur, which may be due to limitations of the method itself. Because of the relatively low molar masses of the polyester fractions, a swollen, gel-like polymer-rich phase is formed, rather than a distinct solid precipitate. Thus, for low \%-solvent, it was difficult to obtain a non-turbid supernatant phase Furthermore, due to low concentrations, the relative influence of the injection solvent in the HPLC measurements, which were carried out in the SEC

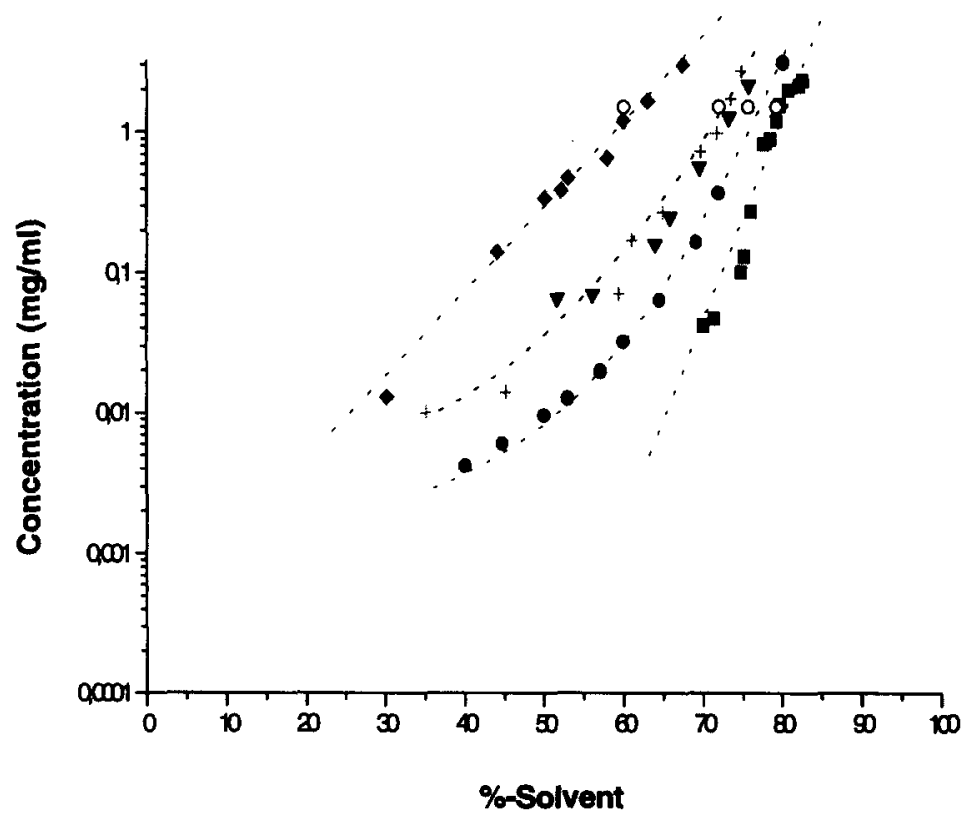

Fig. 5. Maximum solubility of several polyester fractions versus solven/non-solvent composition at $21^{\circ} \mathrm{C}$. (ם) Fraction 2 ; ( $(\boldsymbol{\theta})$ fraction 3 ; $(+)$ fraction $4 ;(\nabla)$ fraction $4(2)$; $(\$)$ fraction $6 ;(O)$ cloud point measurement. 
mode, increased, thus complicating an accurate quantification. For fraction 4, measurements were carried out twice with a time difference of 6 months, using freshly obtained fractions. As can be observed from Fig. 5, reproducibility is satisfactory for our purposes.

It is also interesting to note that CPC values of the respective fractions obtained by cloud point titrations fit well in the solubility curves (open symbols in Fig. $5)$. This confirms the reliability of the used titration method despite of low sample amounts and visual observation of the cloud points.

For decreasing molar masses, a more gradual increase of solubility with \%-solvent is obtained which is also predicted by Eq. (1). The observed strong concentration dependences explain the broad peaks obtained on a glass column, where separation is only governed by solubility effects (Fig. 2). Furthermore, the steeper lines for higher-molar-mass fractions in Fig. 5 can also be recognized in Fig. 2, where peak width decreases with increasing molar mass.

In Fig. 6, solubility measurements for fractions 2, 4 and 6 are compared with concentrations eluting from the glass column. Since known amounts were injected, the eluting masses at respective $\%$-solvent compositions could be calculated from the fractional peak areas, after correction for breakthrough. Hereto, slice widths of $0.5 \mathrm{~min}$ were taken. By dividing eluting mass by the volumetric slice width $(0.5$ $\min \cdot F$ ), eluting concentrations were obtained.

As can be observed, concentration profiles obtained from chromatographic measurements do not coincide with the curves of maximum solubility. For the high \%-solvent part of the distribution this is trivial, since the available mass gets exhausted, which is represented by a final decrease in concentration in the eluate. However, for the low \%solvent part this is remarkable, since this part represents the beginning of the gradient elution experiment, where enough mass is available to obtain saturated solutions!

The observed "elution delay" cannot be the result of sorption effects, as has been shown earlier. Furthermore, small errors in the system hold-up volume, which is necessary to calculate the \%-solvent at each elution time provide by no means an explanation for this phenomenon. Therefore, the eluate indeed is not saturated, indicating that no thermodynamic equilibrium was reached during redissolution which is probably due to kinetic effects.

In order to confirm this assumption, several practi-

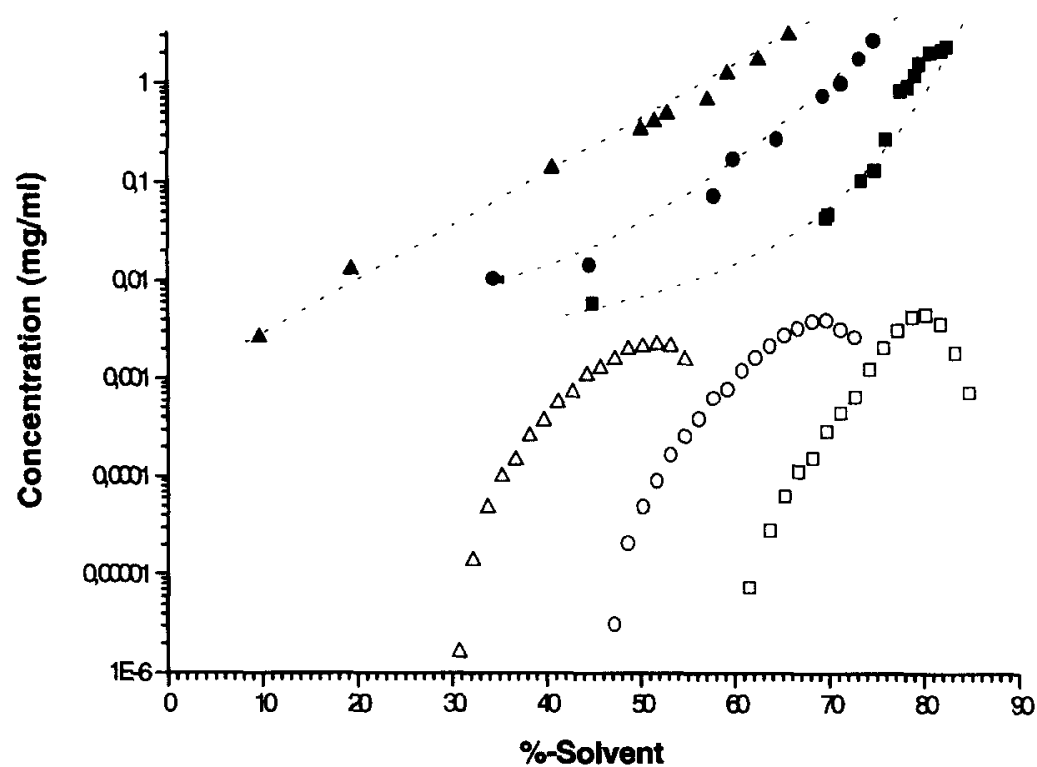

Fig. 6. Comparison of solubility measurements under static conditions (filled symbols) with concentrations eluting from the glass column (open symbols) at $21^{\circ} \mathrm{C} .(\square)$ Fraction $2 ;(\bigcirc)$ fraction $4 ;(\triangle)$ fraction 6. GPEC conditions: see Fig. 2. 
cal parameters which can influence re-dissolution kinetics, e.g., temperature and gradient steepness were varied. For reasons of available amounts, fraction 3 was used for these experiments.

From Fig. 7a it can be observed that a temperature increase gives rise to higher concentrations of the eluting polyester, at a fixed \%-solvent. This is due to increased solubility of the polyester in the mobile phase causing earlier elution, which is a thermodynamic rather than a kinetic effect. The curve of maximum solubility at these temperatures, which was not measured for reasons of practical difficulties, would, however, also shift to higher concentrations. It is therefore obvious, that even at $60^{\circ} \mathrm{C}$, which is about the highest temperature that can practically be applied for the chosen S/NS system, the influence of re-dissolution kinetics cannot be avoided.

A decrease of gradient steepness causes the end of the distribution to elute at somewhat lower \%-solvent (Fig. 7b), which is an indication for the importance of re-dissolution kinetics. The concentration differences at low \%-solvent, however, are within experimental error and it is obvious that a decrease of steepness to $1 \% / \mathrm{min}$, which is frequently used in practice [8], does not avoid kinetic effects.

Finally, the increase of the injected amount causes an increase of the eluting concentrations (Fig. 7c), which is another result proving that the eluate is not saturated! Furthermore, a slight shift of the concentration maximum towards higher \%-solvent can be observed, which confirms the importance of redissolution kinetics.

Although the changes in elution behaviour, with changing experimental parameters are significant, the observed effects are rather small. This might be expected, since the dependence between kinetic effects and parameters such as temperature are generally described by exponential functions [32]. Therefore, for a further verification of re-dissolution kinetics, a more pronounced change in practical parameters by one or more decades, would be necessary. Since practical parameters in chromatography, e.g., temperature and flow-rate can only be varied within small limits this cannot be realized.

Although it is obvious that re-dissolution is influenced by kinetic effects, these effects apparently do not affect the separation on a $\mathrm{C}_{18}$ column. Obviously, after time-dependent re-dissolution, adhe- sion forces are replaced by sorption forces, ensuring normal retention behaviour, governed by sorption effects. It is imaginable, however, that the separation on a less retaining column, for instance a silica derivatized cyanopropyl phase, could indeed be dominated by re-dissolution effects. Especially for the high-molar-mass part this might be expected since it has been pointed out in this study that the retention difference between a non-retaining column and a $\mathrm{C}_{18}$ column is already very small! This difference would even be smaller, or non-existing when a less retaining column is be used.

The effect of re-dissolution kinetics influencing polymer separations has also been observed by other workers $[19,20,23]$. Furthermore, in a future study we will clearly show re-dissolution effects influencing the separation of crystalline polyesters, even when a $\mathrm{C}_{18}$ column is used [21]!

\section{Conclusions}

Solubility effects in GPEC under reversed-phase conditions of polyester resins using $\mathrm{THF}$-water as S/NS combination, were investigated using low polydispersity fractions obtained by SEC. Due to pronounced dependencies of molar mass and concentration on cloud points, these effects can at best be studied under chromatographic conditions, using inert column packings. Bare silica, non-porous glass and a stainless steel precolumn filter were compared for the use as an inert medium. The use of glass was shown to be the best choice in this respect. Bare silica causes additional retention which is probably caused by hydrophobic effects. A precolumn filter gives rise to problems due to breakthrough, which is due to a reduced surface area available for precipitation, and insufficient redissolution. By comparison with the results on the inert glass column, the separation on $\mathrm{C}_{18}$ throughout the whole investigated molar mass range was shown to be solely determined by sorption effects. The observed correspondence of $\phi_{\mathrm{e}}$ on $\mathrm{C}_{18}$ and glass for the high-molar-mass fractions can be explained by the fact that, due to low values of $K_{\mathrm{av}}$, the distribution in the stationary phase will be minor, after the retention factor has decreased below a certain value. This point is in reversed-phase 


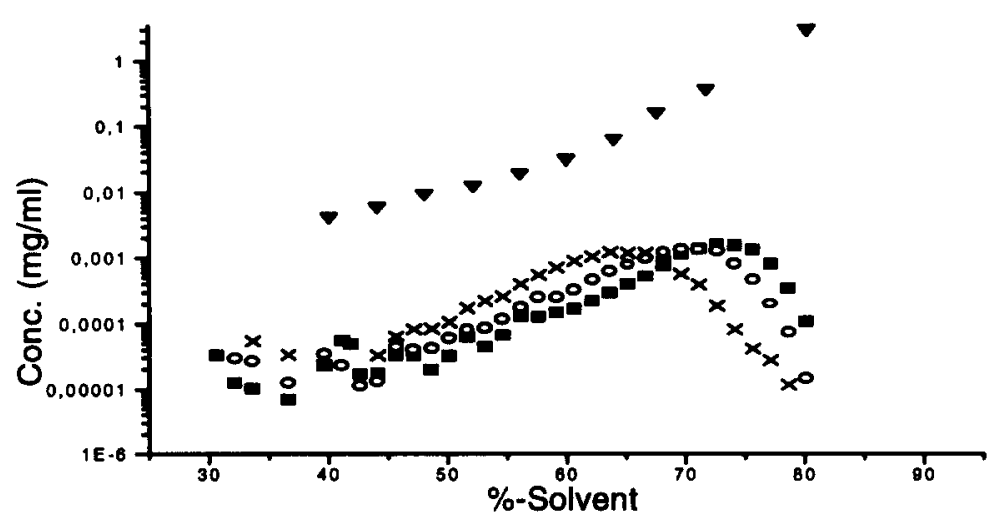

a)

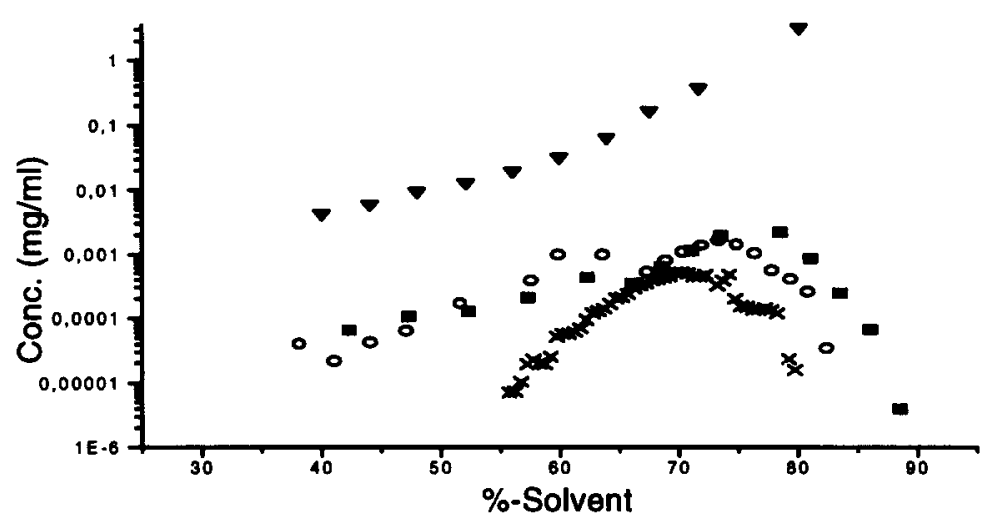

b)

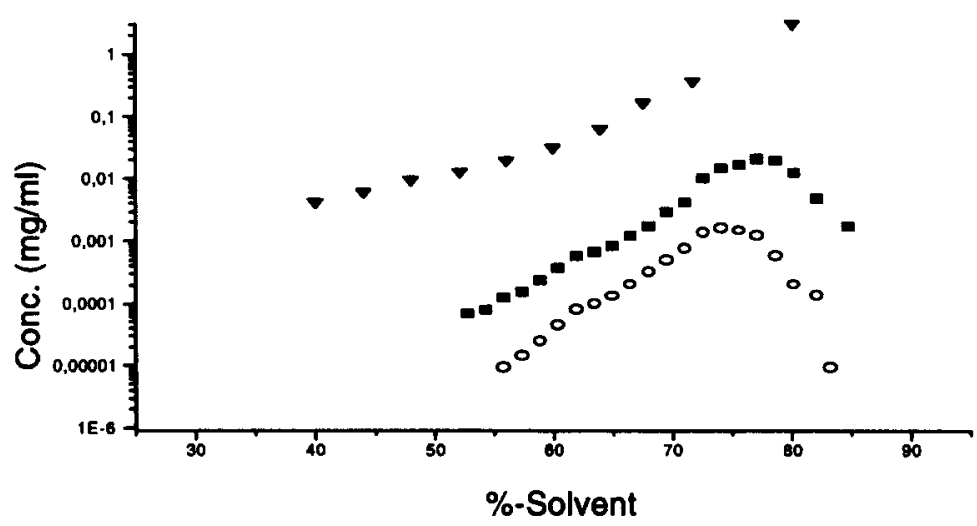

c)

Fig. 7. Influence of temperature (a), gradient steepness (b) and injected amount (c) on the eluting concentrations of polyester fraction 3.

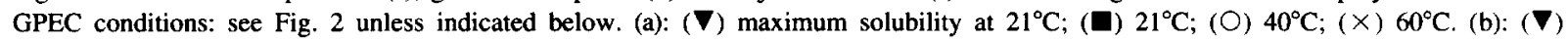

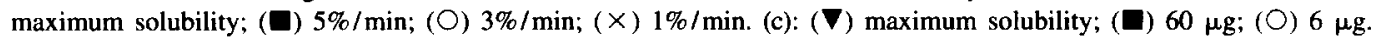


systems mainly determined by interactions between sample and mobile phase, which are the same interactions that determine solubility. The finding of corresponding values of $\phi_{\mathrm{c}}$ on an inert and a sorbing column of the whole, unfractionated polyester alone, is therefore no evidence for solubility governing retention in the high-molar-mass part of the chromatogram.

A comparison with measurements of maximum solubility under static equilibrium conditions of four different polyester fractions in various S/NS combinations revealed that concentrations of the eluting fractions on the glass column are considerably lower than maximum solubility. This can only be explained by redissolution kinetics. The contribution of kinetic effects was confirmed by the effect of temperature and gradient steepness on the elution behaviour, although the observed changes were rather small. Re-dissolution kinetics do not influence the separation of the investigated polyester on $C_{18}$. This, however, is not the case for all types of polyesters, as will be pointed out in a future study.

\section{Acknowledgments}

The authors greatly acknowledge Dr. Eric Nies for his useful comments. We further thank Mr. Richard Wolters for performing part of the experimental work during his graduation project and Mr. Ber Vermeer for his technical assistance.

\section{References}

[1] W.J. Staal, P. Cools, A.M. van Herk and A.L. German, J. Liq. Chromatogr., 17 (1994) 3191.

[2] P.J.C.H. Cools, A.M. van Herk, A.L. German and W.J. Staal, J. Liq. Chromatogr., 17 (1994) 3133.

[3] G. Glöckner, H. Kroschwitz and C. Meissner, Acta Polymerica, 33 (1982) 614.

[4] S. Mori, Anal. Chem., 62 (1990) 1902.

[5] G.H.J. van Doremaele, H.A. Claessens and A.L. German, Chromatographia, 31 (1991) 493.

[6] S. Tanaka, M. Uno, S. Teremachi and Y. Tsukahara, Polymer, 36 (1995) 2219.
[7] P.J.C.H. Cools, F. Maessen, B. Klumperman, A.M. van Herk and A.L. German, J. Chromatogr. A, 736 (1996) 125.

[8] H.J.A. Philipsen, B. Klumperman and A.L. German, J. Chromatogr. A, 746 (1996) 211.

[9] P. Kilz, R.P. Krüger, H. Much, G. Schulz, in T. Provder, H.G. Barth and M.W. Urban (Editors), Chromatographic Characterization of Polymers - Hyphenated and Multidimensional Techniques, American Chemical Society, Washington, DC, 1995, p. 223.

[10] S. Podzimek and J. Hyrsl, J. Appl. Polym. Sci., 53 (1994) 1351 .

[11] T.C. Schunk, J. Chromatogr. A, 656 (1993) 591.

[12] G. Glöckner, Chromatographia, 25 (1988) 854.

[13] G. Glöckner and D. Wolf, Chromatographia, 34 (1992) 363.

[14] G. Glöckner and H.G. Barth, J. Chromatogr., 499 (1990) 645.

[15] R. Schultz and H. Engelhardt, Chromatographia, 29 (1990) 205.

[16] G. Glöckner, Gradient HPLC of Copolymers and Chromatographic Cross-fractionation, Springer Verlag, Berlin, Heidelberg, New York, 1991.

[17] G. Glöckner, Z. Phys. Chem., 229 (1965) 98.

[18] G. Glöckner, Chromatographia, 37 (1993) 7.

[19] G. Glöckner, D. Wolf and H. Engelhardt, Chromatographia, 38 (1994) 749.

[20] G. Glöckner, D. Wolf and H. Engelhardt, Chromatographia, 39 (1994) 557.

[21] H.J.A. Philipsen, M. Oestreich, B. Klumperman and A.L. German, in preparation.

[22] M.R. de Cooker, Study to the Influence of Solubility Effects in GPEC of Polyesters, using Narrow Disperse Standards, Obtained by Fractionation Methods, MSc. Thesis, 1995, Eindhoven University of Technology.

[23] R.A. Shalliker, P.E. Kavanagh and I.M. Russell, J. Chromatogr., 543 ( 1991 ) 157.

[24] E.F. Casassa, in L.H.Tung (Editor), Fractionation of Synthetic Polymers: Principles and Practices, Marcel Dekker, New York, 1977, chp. 1.

[25] G. Glöckner, J. Appl. Polym. Sci., 43 (1989) 39.

[26] R. Schultz and H. Engelhardt, Chromatographia, 29 (1990) 325.

[27] P. Hambleton, W.J. Lough, J. Maltas and M.J. Mills, J. Liq. Chromatogr,, 18 (1995) 3205.

[28] C.H. Lochmuller and M.B. McGranaghan, Anal. Chem., 61 (1989) 2449.

[29] M.A. Quarry, M.A. Stadalius, T.H. Mourey and L.R. Snyder, J. Chromatogr., 358 (1986) 1.

[30] M.A. Stadalius, M.A. Quarry, T.H. Mourey and L.R. Snyder, J. Chromatogr., 358 (1986) 17.

[31] L.R. Snyder, J.L. Glajch and J.J. Kirkland, Practical HPLC Method Development, Wiley, New York, 1988.

[32] W.J. Moore, Physical Chemistry, Longman Group Limited, London, 1972. 